\title{
Modeling Contexts for Business Process Oriented Knowledge Support
}

\author{
Marielba Zacarias ${ }^{1,2}$, Artur Caetano ${ }^{1,3}$, Sofia Pinto ${ }^{3,4}$ José Tribolet $^{1,3}$ \\ 1 Center for Organizational Engineering, INESC, Rua Alves Redol 9, 1000-029 Lisboa, Portugal. \\ 2 Universidade do Algarve, ADEEC-FCT, Faro, Portugal. \\ 3 Department of Information Systems and Computer Science, Instituto Superior Técnico, \\ Technical University of Lisbon, Portugal. \\ 4 ALGOS, INESC-ID, Rua Alves Redol 9, 1000-029 Lisboa, Portugal \\ mzacaria@ualg.pt, artur.caetano@inov.pt, sofia@algos.inesc-id.pt, jose.tribolet@inov.pt
}

\begin{abstract}
While performing knowledge driven tasks, people at organizations spend time searching the required support information. In order to effectively support knowledge driven tasks, information should be timely and proactively provided to its consumers according to its usage context and patterns. In this paper we define the concepts of action and interaction contexts. More specifically, we define action context as the interplay of actor, role, task and time related features. We focus on actors' capabilities for handling several action contexts. Interaction contexts are defined as the supporting components of actors' interactions related to business processes. Actors and business processes are approached as a network of action and interaction contexts managed by an "operating system". We relate the defined concepts and propose a business process oriented and context-based modeling approach which aims to provide a richer, efficient and flexible semantics, capable of better representing the complexity of actual business process execution and facilitating a proactive and timely knowledge support of business actors. We illustrate some of these ideas with a working example.
\end{abstract}

\section{Introduction}

The highly dynamic nature of today's organizations continually presses for faster ways of obtaining the information required for their operation. Despite the number of tools and systems developed to collect, organize and disseminate the information created throughout the organization, people at work continue to spend time searching the information required for their activities. This situation is aggravated in knowledgedriven tasks, which have greater information and collaboration requirements. When accomplishing business activities, knowledge consumers should be able to receive information according to their needs. Further, knowledge provision activities should minimize the disturbance of workers' core activities. In order to meet these requirements, timely and proactive knowledge distribution mechanisms should be devised.

Current knowledge management systems offer limited solutions to the problem of knowledge provision and are frequently deserted by users [2]. Recent research projects [4,12] are recognizing the social, contextual and heterogeneous nature of knowledge sources, and are promoting a distributed approach to knowledge management. Theoretical frameworks and technical solutions are being developed aiming to provide a more appropriate support of knowledge-related processes. However, these developments are not business processoriented and do not focus the dynamics of individuals' behavior changing at work.

Both people and business processes are business actors capable of performing several tasks and roles. Therefore, business actors exhibit different behaviors with different information requirements. These requirements depend on a number of factors such as individual features, task at hand and role played. A timely and proactive information provision entails considering not only actor, role and task-related features. It must also take into account the dynamics that governs task changing behavior. Capturing these dynamics requires (1) a different organizational perspective, (2) defining new business concepts and (3) adjusting existing ones to accommodate newly defined concepts.

Business models allow organizations to communicate, document and understand its activity [5]. In order to manage organization's complexity, business models use a number of different perspectives. A business process perspective of organizations implies relating a set of activities in sequences that deliver some value to internal or external customers. Modeling business processes involves capturing interactions between multiple business objects, such as activities, goals, resources and human or automated actors [6]. Workflow management systems are a supporting technology for business process modeling, optimization and automation 
[7]. Since the semantics of business process models and workflows are similar, workflow schemas can be directly derived from business process models [8]. As workflows enable information dissemination among users and systems, they also facilitate a "process oriented" knowledge management [9]. Nevertheless, a process-oriented knowledge support through workflows has two limitations. On one side, conventional business process models and workflows support planned and predefined work, offering little flexibility. Alternative approaches such as adaptive and agent-based workflows are enhancing workflow's flexibility.

On the other side, conventional business process models and workflows do not capture business actors' changing behavior patterns. They describe actors as uniform units with a single behavior. This forces the representation of actors' different behaviors as independent and unrelated units. Role based modeling $[1,5,6]$ overcomes this limitation by enabling the representation of different "views" of a single actor. This approach addresses static aspects of actors' multiple behaviors. In order to give business actors an overall and customized knowledge support, it is necessary to address the dynamic aspects of behavior changing. This entails capturing actors' engagement and disengagement patterns to tasks and roles.

In this paper we define the concepts of action and interaction contexts as key elements of a business process oriented modeling approach which aims to provide a richer, efficient and flexible semantics, capable of better representing the complexity of actual business process execution and facilitating a proactive and timely knowledge support to business actors. The remaining of this paper is structured as follows: section 2 reviews related work on context and role-based modeling, section 3 defines the core concepts of this work, section 4 presents the proposed modeling approach. Section 5 illustrates some of these ideas. In section 6, we give our conclusions and future directions.

\section{Related work}

This section presents related work supporting our modeling approach. In section 2.1, the concept of action context is based on formalization notions coming from research on theories of context. One context element, the role, is based on the role-based business process modeling approach described in section 2.2.

\subsection{The notion and uses of context}

Although playing an important role in multiple disciplines such as pragmatics, natural language semantics, linguistics, cognitive psychology, and artificial intelligence [10], there is no standard concept or theory about context. The notion of context varies according its area of application. However, there is consensus around the relational nature of context i.e. context it is not an autonomous entity, is always related to something else. From an engineering perspective, context is viewed a collection of things (sentences, propositions, assumptions, properties, procedures, rules, facts, concepts, constraints, sentences, etc) associated to some specific situation (environment, domain, task, agents, interactions, conversations, etc). This consensus is reflected by the "box metaphor" [11]. The intuition is that context can then be seen as a container where its content depends on some set of situational characteristics or parameters (figure 1).

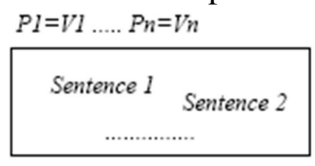

Fig. 1. The "Box Metaphor" [11]

The specific set of parameters varies according to areas of application. In pragmatics, indexical expressions are defined as expressions dependent on indexes such as place, time, agent and world, which are a subset of context parameters. In Artificial Intelligence, parameters (called dimensions) such as time, location, culture, topic, granularity and modality among others, have been proposed as defining elements of context space [13]. A proposal for a workflow context space in [14] includes the following parameters; function, behavior, causality, organization, information, operation and history. In the area of context aware applications the localization, user identity, activity and time has been identified parameters of context [15]. In terms of its use, context is typically employed in two broad different ways. On one hand, context has been used as a 
partitioning or grouping means. This kind of context use has two different purposes: (a) enabling filtering, classification or customization mechanisms and (b) achieving representation or reasoning efficiency. On the other hand, it has been employed as a means for making explicit assumptions, interpretations or concepts, to resolve interoperability problems derived from heterogeneous information or data interchange.

\subsection{Role based Business process modeling}

Conventional business process models describe actors and other business objects with a pre-defined and uniform behavior. This leads to the representation of business objects capable of exhibiting multiple behaviors as independent and unrelated units. Role based Business process modeling [1,5,6] overcomes this limitation by enabling the partition of business objects behavior according its relationships with other business objects. Business objects interact and relate with other business objects and play roles for each other during these collaborations [5,6]. For this reason, the semantics of a business object depends on the information extracted from its relationships and collaborations. The overall motivation for role-based business process modeling is allowing specific views on business objects. These views are used by other business objects as a means to improve knowledge over the object's capabilities, allowing them to selective access the object. A view is a set of selected properties. An important feature of these views is that they can change dynamically, i.e. be added or removed from a business object. Figure 2 illustrates how role-based business process modeling enables several views of business objects.

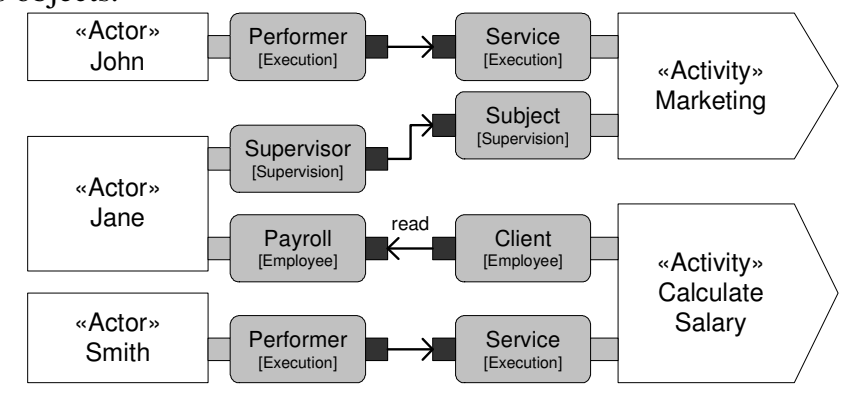

Fig. 2. Role-based modeling example [6]

Each one of these views is modeled as a role. A role defines the properties of the business object that are relevant when interacting with other business objects, thus defining a part of its observable behavior. The union of all roles that a business object is able to play defines its complete observable behavior. This example shows how a business object may behave differently depending on its usage context. The business object Jane, which is modeled as an actor and plays a supervisor role in its collaboration with the marketing activity, is related to the calculate salary activity via the employee role. This role defines the specific business properties that are required for an actor to have so that its corresponding salary may be computed.

\section{Action and interaction contexts}

The present section presents the core concepts of our approach. The concepts of action and interaction contexts focus the multi-tasking nature of business actors and their capability to handle several contexts. Knowledge flows among actors are approached establishing analogies with distributed systems. Interaction contexts are the supporting element of these flows.

\subsection{Actors' multi-tasking behavior}

Human beings by nature are capable of alternating among several, independent tasks. When engaged in several activities, people "break" these activities and "jump" among them according to criteria such as task priorities, task resource's availability or scheduling-related habits (ex. hour preferences, dispatching shorter tasks first, etc.). Human multi-tasking capabilities and its limitations are subject of research in Experimental 
Psychology [18]. Several theories from this area support the existence of mental executive control processes that supervise the selection, initiation, execution, and termination of tasks. An analogy of these executive processes with computational multi-tasking operating systems has been established in [19]. Understanding executive mental control may help to understand and describe the dynamics of human actors' behavior changing. Executive mental control defines human actors' basic operative behavior. Although exhibiting distinct behaviors with different information requirements, business actors are unique entities. Hence, an overall actors' support entails capturing not only its specific behaviors, but also his basic operative behavior.

\subsection{The action context concept}

The context of a knowledge worker's information needs is determined by three main factors: (1) the individual person, (2) his/her position in the organizational structure, (3) the task at hand [3]. Taking into account all three dimensions promises better results than concentrating on any subset. From our point view, information needs are determined by smaller units of behaviors determined by action contexts. Each specific business actor, role and task combination determines a different action context, where the concept of role is approached as described in section 2.2.

Action contexts define relevant information and behavior for a specific individual, role and task. As we consider time a defining factor of relevance, action contexts include a temporal dimension. In terms of the box metaphor, action contexts parameters are individual, role, role, task and time related features and its contents are the collection of relevant information and behavior items corresponding to a specific set of parameters values. Actors' behaviors and requirements are typically defined according to task or role-related features. By adding specific individual's-related-features -such as the degree of expertise on a task -, we enhance customization possibilities to actor' representation. Figure 3 illustrates the defining features of action contexts.

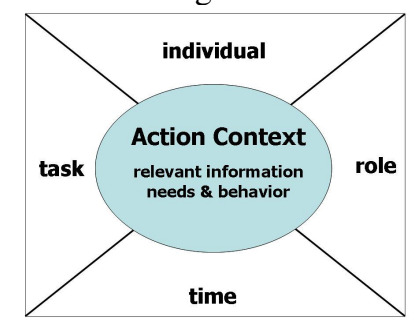

Fig. 3. The concept of action context

Action contexts and the concept of knowledge. Despite the consensus around the existence of a continuum of data-information-knowledge, the limits among these concepts are still not clear. The context dependence of knowledge has been largely recognized. The borderlines between data, information and knowledge are not sharp because they are relative with respect of the context of use [16]. In this work, action contexts are approached as active and dynamic entities that can be seen as "running processes" representing specific actors involved in a specific task, role during a time interval. This approach entails a dynamic determination of relevance which enables more powerful and flexible representations of information needs than pre-defined sets of relevant items. In this work, all action contexts' relevant items are considered knowledge items. Thus, knowledge is produced and consumed in specific action contexts and its meaning is relative to these action contexts.

\subsection{The interaction context}

By accepting the multi-tasking capabilities of business actors, the dynamics of theirs' interactions can be approached establishing analogies with distributed systems. Interactions entail a communication process. Distributed systems' communication can be modeled as the information transfer from sender process and a receiver process. This information transfer is supported by a channel. According to Communication Theory [21], this channel entails the use of a sign system. Sign systems are described by a layered model integrated by four interdependent levels; empirics, syntactics, semantics and pragmatics. The ISO/OSI model provides the standard communication channel for distributed computational systems, including Unix sockets, application integration's middleware, agents' and web services' communication models, with varying degrees of 
sophistication offered by the layers over the transport layer [23]. The ISO/OSI model addresses empirical and syntactical layers. Advances in Ontologies' research, are providing higher level syntactics and semantics to communication among automated actors such as agents and web-services [22,24]. The inclusion of interaction protocols and roles has brought pragmatic-related elements.

In this work, we focus the "communication channel" of business activities. Each business activity in execution originates a communication channel defined as interaction contexts. Interaction contexts provide a shared empiric, syntactic, semantic and pragmatic space for actors' communication related to business activities. Business activities provide the business logic, i.e. activity-related ontology, activity history, interaction protocols, rules and role structures into interaction contexts. Interaction contexts complement business logic with knowledge distribution processes such as document and interaction management, directory and routing services, coordination and negotiation services. While computational distributed systems implement these services through specialized software components, in human environments, these services are implemented by the interacting human actors themselves. In this case, human actors operate both at the individual level and as active elements of the interaction context. Including interaction contexts as an active and defining component of actors' interactions enhances the semantics of actors' interactions. Further, aspects not belonging to any particular actor but to the interactions among them can be efficiently represented in one single collective concept.

\subsection{Approaching business actors as networks}

The human cognitive and perceptual systems are designed to identify and use context automatically as we go about our daily lives [17]. Due to their multi-tasking behavior, actors' are capable of handling several action contexts. But, similarly to automated actors, scarce resources such as attention and short-term memory [20], forces the activation of only one action context at once. At some specific time frame, actors' information requirements depend of the active action context. Thus, when modeling actors, it should be considered the existence of various action contexts for each actor, and the possibility of switching among them.

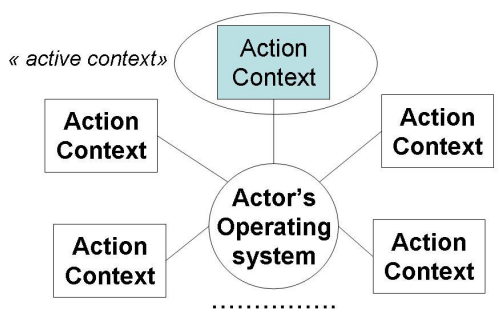

Fig. 4. Modeling multi-tasking actors

Figure 4 illustrates our proposed approach for modeling actors. Modeling actors' multi-tasking behavior entails "exploding" the notion of actor. Rather than considering an actor as a single object, we are proposing modeling actors as a network of objects: the action contexts. Action contexts are not autonomous, as they are managed by a different and special object: the actors' operating system. Actors' operating system handles actors' general synchronization and scheduling capabilities such as action context initiation, termination and switching mechanisms. This approach offers a richer semantics that allows an overall support of actors' information needs. It also efficient as it allows the representation of actor's basic behavior in one single element.

As we seek a business process perspective, in this work the aim is to capture knowledge flows among actors related to business processes execution i.e., that take place within the context of some business activities. The execution of a business activity originates an interaction context which is initiated, suspended or terminated by the business process management system (BPMS). BPMS are considered a special kind of actor that mediates other actors' interactions and are approached as a network of interaction contexts managed by the business process "operating system". Under this approach, business process' scheduling, may be captured and represented through more sophisticated scheduling algorithms or rules, enhancing the business process model's flexibility. 


\section{A business process-oriented, context-based model}

Figure 5 illustrates the proposed model for business actors and their contexts. In first place, two kind of actors are defined; individual actors and process-related actors. Individual actors represent human or automated actors that may be involved in several activities and tasks belonging to different business processes. Process-related actors are also human or automated business actors, operating in behalf of a business process. Interactions among individual actors are regulated and supported by process-related actors.

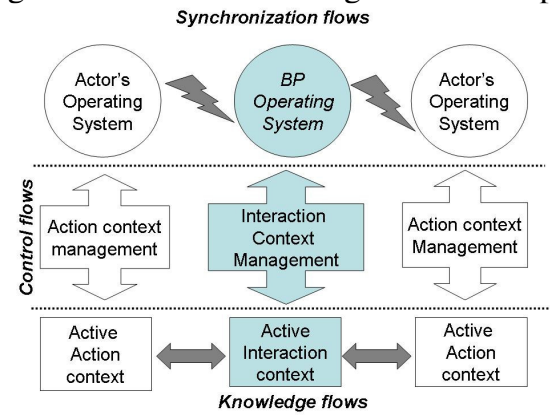

Fig. 5. Modeling business actors and contexts

In second place, the model has three layers; interaction, control and synchronization. Knowledge production entails "synchronizing" with the corresponding business process' operating system, which will route the produced item to the appropriate interaction context. The interaction context identifies the receivers and distributes the item to them. In order to receive an item, actors' operating systems "synchronize" with the business process and determine the item's relevance to their action contexts. Actual knowledge consumption takes places, once the receiver's operating system activates the corresponding action context. Action context management operations realized by individual actors' operating systems determine actual actors' schedule. Interaction context management operations are realized by process' operating system and determine the actual scheduling of business activities. These operations are represented in the control layer. The interactions that enable knowledge flows occur at the interaction layer. Knowledge production and consumption occurs when actors are located in specific action contexts. Knowledge distribution is realized in the interaction context.

\section{Modeling Examples}

In order to illustrate some of the previous ideas, in this section we give a modeling example based on observations made on a real organizational setting. Alice is an assistant to the presidency of a research institute. Figure 6 shows a role-based model of three different behaviors of Alice, each one determined by Alice's engagement in three activities; (1) in activity Elaborate Student's List playing the role supervisor,(2) in activity Assist Professor playing the role of performer and (3) in activity Elaborate Budget playing the Training Role. The role-based model of figure 6 offers a static model of Alice's different behaviors.

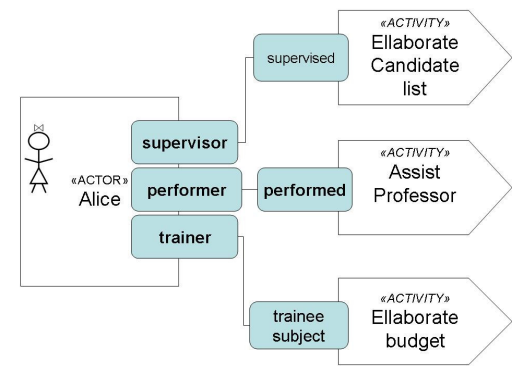

Fig. 6. Alice's role-based model

Modeling actors' changing behavior. Figure 7a illustrates one scenario of Alice's behavior changing. The figure shows a sequence of action context switches and shows, her action context switching rules. While 
specific requirements and behaviors are captured by action contexts, capturing behavior changing patterns allows supporting overall actors' requirements and anticipating specific requirements and providing them proactively. Although figure 7 describes one particular scenario, lasting patterns of task-switching behaviors should be captured, since only these are susceptible of systems support.

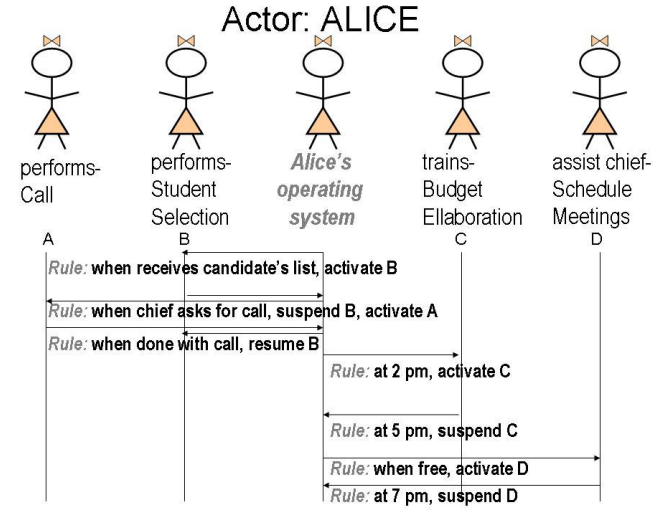

Fig. 7a. Modeling actors

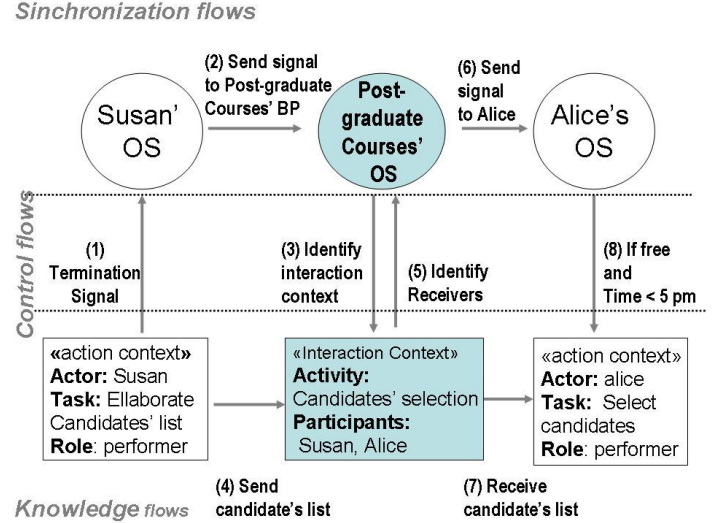

Fig. 7b. Modeling flows among actors

Modeling flows among actors. Figure $7 \mathrm{~b}$ illustrates the model described in section 4. It models a knowledge flow scenario among two actors due to the Post-graduate Course's business process execution based on the observation: "When Susan finishes the candidate list, sends a mail to Alice attaching the list. Alice receives the mail and decides to select students if finishing her activities before $5 \mathrm{pm}$ ". Figure 8 explicitly shows the synchronization and control flows involved when the knowledge item Candidate List is transferred from its producer (action context Susan-performs-Ellaborate candidate list) to its consumer (action context AlicePerforms-Students Selection). It also shows the action context's activating rule. This example describes an interaction scenario of two human actors and a non-automated business process. Flows 1-6 are originated by Susan. She acts as an individual actor when elaborating the list. Since sending the list to Alice entails knowing (1) the list is a resource of the POSI business process' resource and (2) the receivers of the list, she also acts as a process-related actor. Flows 7-9 are originated by Alice acting as an individual actor. Upon the reception of the mail, she will identify the proper action context and will activate according to her own switching rules.

\section{Conclusions and future work}

In this work the concepts of action and interaction contexts are defined as key elements in modeling business actors and the knowledge flows among them. By focusing on business actors' multi-tasking capabilities, business actors and their interactions are approached as a network of action and interaction contexts. The proposed model -based on analogies with multi-tasking operating systems and distributed systems' communication model-, separates synchronization and control flows from actual knowledge flows. Two kind of business actors are distinguished; individual and process-related actors.

Representing actors as a network of action contexts managed by an operating system seeks a richer semantics that allows an overall support of actors' knowledge production and consumption issues. Further, placing general behavior in one single element avoids duplication and thus, offers a more efficient representation. Representing business processes as a network of interaction contexts managed by an operating system enhances flexibility of business process models. The inclusion of interaction contexts seeks a richer semantics for knowledge distribution issues. It also offers an efficient representation, by allowing to model aspects not belonging to any particular actor but to the interactions among them, in one single collective element. The three layers of flows aims to handle the complexity of business actors and their interactions, related to their multi-tasking behavior. By distinguishing individual aspects from business process-driven aspects, allows a flexible and efficient modeling of actor-process collaborations. Finally, the model allows an integrated view of knowledge-related processes with business processes, facilitating a business process oriented, proactive and timely knowledge support to business actors.

The concepts defined and proposed modeling approach are part of a more comprehensive research which aims to the provision of a rich, flexible and efficient semantics, capable of better representing the complexity 
of business actors and business processes. The modeling examples offer simple and limited views that illustrate some aspects of our proposal. An extensive modeling work is still required in order to test, adjust and refine the concepts presented. Research focusing on specific model elements will lead to more precise and formal descriptions of their relevant properties. Model evaluation will be conducted through business cases.

\section{References}

[1] M. Ould, "Business Processes, Modeling and Analysis for Reengineering and Improvement", John Wiley \& Sons, 1995.

[2] M. Bonifacio, P. Bouquet, Distributed Knowledge Management: a Systemic Approach, Gianfranco Minati, Eliano Pessa (Eds.), Emergence in Complex, Cognitive, Social and Biological Systems, Kluwer Academic / Plenum Publishers, New York, 2002

[3] Van Elst L., Becker A., Maus H., "Exploiting User and Process Context for Knowledge Management Systems". Workshop on User-Modeling for Context Aware Aplications. $8^{\text {th }}$ International Conference on User Modeling, July 2001, Germany

[4] The EDAMOK project http://www. Edamok.itc.it

[5] Caetano A., Silva R., Tribolet J., "Business Process Modeling with Objects and Roles". Proceedings of the 6th International Conference on Enterprise Information Systems (ICEIS 2004), Porto, Portugal, April 14-17, 2004, pp. 204-209.

[6] Caetano A., Silva A., Tribolet J, "Object-Oriented Business Process Modeling with Roles". 7th International Conference on Information Systems Implementation Modeling (ISIM 04), Czech Republic, 2004

[7] Georgakopoulos D. Hornick M. "An Overview of Workflow Management: From Process Modeling to Workflow Automation Infrastructure”, Distributed and Parallel Databases, 3:119-153, Kluwer Academic Publishers, Boston, 1995.

[8] Aalst W., Hee K., "Workflow management models, methods and Systems", MIT Press, 2002.

[9] Jablonski S., Horn S., Schlundt M., "Process Oriented Knowledge Management" 1. Konferenz Professionelles Wissensmanagement: Erfahrungen und Visionen, Baden-Baden, 2001 (mit S. Horn, M. Schlundt).

[10] Bouquet P., Ghidini C., Giunchiglia F., Blanzieri E., "Theories and Uses of Context in Knowledge Representation and Reasoning", Journal of pragmatics - Special issue on context, 2002

[11] Benerecetti M., Bouquet P., Ghidini C.," On the dimensions of context dependence: partiality, approximation, and perspective". Third International and Interdisciplinary Conference on Modelling and Using Context (CONTEXT'01). Scotland, 27-30 July, 2001. Springer-Verlag, Vol. 2116, p. 59-72,. Akman V., Bouquet P., Thomason R., Young R. A., Dundee (eds.)

[12] The SWAP Project http://www.swap.semanticweb.org

[13] LENAT D., The Dimensions of Context-Space, CycCorp, October 28, 1998 [online - 03/08/03], http://casbah.org/resources/cycContextSpace.shtml

[14] Maus H. "Workflow Context as a Means for Intelligent Information Support" Third International and Interdisciplinary Conference on Modeling and Using Context. CONTEXT 2001, Springer-Verlag Berlin Heidelberg, V. Akman et al. (Eds.), LNAI 2116, pp. 261-274, 2001.

[15] Dey A., Abowd G. "Towards a Better Understanding of Context and Context-Awareness". Springer-Verlag, 2001 ISSN:1617-4909, p. 1 -3, Londres, UK

[16] Knowledge Engineering and Management: The CommonKADS Methodology, Schreiber G., Akkermans H., Anjewierden A., De Hoog R., Shadbolt N., Van de Velde W., Wielinga B., The MIT Press, 1999

[17] Degler D., Battle L, Knowledge Management in Pursuit of Performance: the Challenge of Context, Performance Improvement, ISPI, 39 (6), July 2000 [online - 01/09/2003] http://www.ipgems.com/writing/kmcontext.htm

[18] Rubinstein J., Meyer D., Evans J. Executive Control of Cognitive Processes in Task Switching, Journal of Experimental Psychology: Human Perception and Performance August 2001 Vol. 27, No. 4, 763-797., 2001.

[19] Kieras, D. E., Meyer, D. E., Ballas, J. A., \& Lauber, E. J. Modern computational perspectives on executive mental processes and cognitive control: Where to from here? In S. Monsell \& J. Driver (Eds.), Control of Cognitive Processes: Attention and Performance XVIII. Cambridge, MA, MIT Press, 2000

[20] Dix A., Finlay J., Abound G., Beale R., Human-Computer Interaction, Prentice Hall, $2^{\circ}$ Ed., Printed in Great Britain, 1998

[21] Beynon-Davies P., Information Systems: An Introduction to Informatics in Organizations, Palgrave, London, 2002.

[22] Mc.Guiness D., "Ontologies Come of Age". Spinning the Semantic Web: Bringing the World Wide Web to Its Full Potential. MIT Press In Dieter Fensel, J im Hendler, Henry Lieberman, and Wolfgang Wahlster, editors.,2002.

[23] Jeckle M., "Identical Principles, Higher Layers: Modeling Web Services as Protocol Stack" [online - 01/05/04] http://www.jeckle.de/files/xmleu04/

[24] McIlraith S., Son T., Zeng H ., "Semantic Web Services”, IEEE Intelligent Systems, March/April 2001, pp. 46-53 